\title{
Karl Polanyi y la teoría social en América Latina: avances, desarrollos y desafíos actuales
}

\author{
Iván Valenzuela Espinoza \\ Universidad Arturo Prat, Iquique, Chile. \\ Email: leandro.valenzuela@unap.cl
}

\begin{abstract}
Resumen: La riqueza del pensamiento de Karl Polanyi, junto con despertar creciente interés intelectual, suscita distintas y variadas interpretaciones y desarrollos. A continuación se ofrecerá un conjunto de ideas y conceptos derivados de la obra de Polanyi, que están en la base de una emergente propuesta de "teoría social neo-polanyiana” elaborada por importantes autores contemporáneos. El artículo analiza en qué consiste dicha propuesta y cómo contribuye para dar mejor cuenta de diversos procesos y fenómenos del desarrollo en América Latina. Los temas a tratar vienen dados por la relación entre Estado y mercado, la sociedad civil y la innovación institucional, y el "Estado Desarrollista Red".
\end{abstract}

Palabras clave: teoría social, instituciones, desarrollo, Estado.

\section{Karl Polanyi and social theory in Latin America: current developments and challenges}

\begin{abstract}
The richness of Karl Polanyi's sthought attracts growing intellectual interest accompanied by different and varied interpretations and developments. A set of ideas and concepts derived from Karl Polanyi's work will be put forward. It will serve as the basis for an emerging "Neo-Polanyian Social Theory" elaborated by important contemporary authors. The article analyses this proposal and the ways in which it contributes to a better understanding of diverse processes and phenomena in Latin America's development. The issued dealt with include the relations between the State and the economy, civil society and institutional innovation, and the "Developmental Network State".
\end{abstract}

Keywords: Social theory, institutions, development, State.

\section{Karl Polanyi e a teoria social na América Latina: avanços, desenvolvimentos e desafios atuais}

Resumo: A riqueza do pensamento de Karl Polanyi, junto com despertar crescente interesse intelectual, suscita distintas e variadas interpretações e desenvolvimentos. Seguidamente se oferecerá um conjunto de ideias e conceitos derivados da obra de K. Polanyi, e que estão na base de uma emergente proposta de "teoria social neo-polanyiana” elaborada por importantes autores contemporâneos. O artigo analisa em que consiste dita proposta e como ela contribui para esclarecer os diversos processos e fenómenos do desenvolvimento na América Latina. Os temas a tratar vêm dados pela relação entre Estado e mercado, a sociedade civil e a 
inovação institucional, e o “Estado Desenvolvimentista Rede”.

Palavras-chave: teoria social, instituições, desenvolvimento, Estado.

$* * *$

\section{Introducción}

Son numerosos los autores que ponen de relieve el escaso aporte del actual pensamiento económico convencional a la comprensión de numerosos e importantes fenómenos económicos de las tres últimas décadas (ver, por ejemplo, Blyth, 2015). En tono crítico, Santos (2005, 2009), al mismo tiempo, se ha esmerado en desentrañar no sólo las severas limitaciones del pensamiento económico convencional, sino también de parte importante de la teoría social predominante en lo que respecta a los países en vías de desarrollo.

Casi sin contrapeso, los enfoques "neoutilitaristas" propios del "fundamentalismo de mercado" (Block y Somers, 2014) se impusieron en la conceptualización del desarrollo, propiciando la "lógica del intercambio de mercado" y exhortando a la reducción drástica del tamaño del Estado y de su intervención en la economía (Evans, 2007: 35). Dicha orientación puso el acento a más no poder en las supuestas bondades económicas y sociales de los mercados "libres", supeditando drásticamente la vida social y cultural a sus directrices.

Dada la vastedad y complejidad de los fenómenos socioeconómicos y políticos en el mundo y América Latina, se ha tornado imperativo (Santos, 2005; Evans, 2007, 2008b) avanzar en la formulación de marcos conceptuales a tono con la nueva configuración de fenómenos y acontecimientos, los cuales, al mismo tiempo, deberían propiciar visiones alternativas al predominio de las ideas del libre mercado.

Tal como afirma Magali Sarfatti (2015: 59): “En este momento de crisis, tanto del capitalismo como de la ciencia económica que pretende analizarlo, es importante volver a considerar un pensamiento señero como el de Polanyi”. Pues bien, las recientes elaboraciones teóricas de un conjunto de autores, a partir de la obra del pensador socialista húngaro Karl Polanyi (2001 [1944]), ofrecen pistas valiosas para avanzar en la formulación de una robusta teoría social capaz de dar sustento sólido al estudio del desarrollo latinoamericano contemporáneo en ámbitos importantes. Así entonces, sostengo, en lo medular, que la contribución teórica de Polanyi nos ofrece una lúcida y aguda crítica al "fundamentalismo libremercadista" (Block y Somers, 2014: 3), que pone el acento en la "primacía de lo social" frente a la "falacia economicista" (Polanyi, 2001 [1944]: 158-159) que da pie a formulaciones varias de determinismo económico. Asimismo, afirmo que dicha contribución supone un valioso soporte intelectual para revitalizar la teoría social del desarrollo. En tal perspectiva, se pasará revista a diversos 
autores contemporáneos que están impulsando creativamente los recursos intelectuales de la teoría social neo-polanyiana, la cual exhibe un notable potencial para enriquecer los debates latinoamericanos. Al mismo tiempo, los debates en cuestión sin duda que beneficiarán posteriores desarrollos del pensamiento neo-polanyiano.

El artículo consta de cuatro apartados principales. En el primero de ellos serán expuestos los principios e ideas centrales de la teoría social neopolanyiana. Asimismo, en la siguiente sección el foco de atención estará puesto en la reformulación neo-polanyiana del papel del Estado en la economía y la sociedad conforme a la “autonomía arraigada” y la propuesta de Block y Evans. En el tercer apartado, a su vez, se revisará el "giro institucional” en los estudios del desarrollo, así como el alcance de los argumentos neopolanyianos para su reforzamiento teórico. Finalmente, se concluirá con un análisis del llamado "Estado desarrollista red” en tanto que componente clave para las sociedades latinoamericanas en un contexto marcado por la economía global neoliberal.

\section{Principios de la teoría social neo-polanyiana}

Una de las grandes oportunidades que ofrece la revitalización, elaboración crítica y actualización del pensamiento económico y social de Karl Polanyi, radica en su notable potencial para constituirse en un marco teórico adecuado para dar cuenta de forma sofisticada de la economía y los mercados, reconociendo y valorando a la vez la autonomía y lógicas propias de, fundamentalmente, la política, la cultura y el Estado. Simultáneamente, también permite indagar y exponer sus conexiones e interrelaciones en el marco de configuraciones crecientemente complejas ${ }^{1}$.

En palabras del sociólogo Fred Block (2012) uno de los más connotados cultores de la teoría social neo-polanyiana, y de la sociología económica, "la alternativa de Polanyi era retornar a la crítica de la economía política lo que significaba liberar a la gente de la creencia de que la economía debería dictar cómo vivimos nuestras vidas”(p.21-22). Lo anterior guarda relación con refutar la “falacia economicista” (Polanyi 2001 [1944]: 158-159), es decir, la tendencia en pensamiento occidental a concebir todos los aspectos de la existencia social y cultural desde el prisma reduccionista del determinismo económico. Como puntualiza Sarfatti (2015), “sin consideración por la historia, tal falacia supone que el lucro haya sido siempre el motivo determinante de la vida de las sociedades y que la economía de mercado las haya dominado siempre” (p.61)

El argumento de Polanyi, por su parte, consiste en señalar que el abordaje institucional, contrariamente a la ideología del libre mercado, debe asumir plenamente que son las instituciones sociales, políticas y culturales las que modelan y dan forma al funcionamiento de la economía en lugar de las pretendidas “leyes del mercado” (Block y Somers, 2014: 29). 
Conforme a Block y Somers (2014), el pensamiento polanyiano allana el campo del análisis social al distinguir entre la concepción “sustantiva” y la concepción “formal” de la economía. La primera apunta al desafío constante relativo a cómo los seres humanos organizan y asignan los elementos y recursos necesarios para preservar la vida humana, al tiempo que la segunda pone el acento en cómo economizar desde una perspectiva de recursos escasos, lo cual responde a la construcción social e histórica del capitalismo (Ibíd.).

Sobre la base de la distinción establecida, Polanyi (1977) criticó enérgicamente la tendencia economicista que extrapola la maximización económica formal de la economía mercantil a las características y propiedades manifiestamente distintas de las economías no capitalistas (Coraggio, 2009). El pensador húngaro (Polanyi, 1982: 33) sostuvo que, en cambio, la economía sustantiva debe ser entendida en tanto que "proceso institucionalizado de interacción entre el hombre y su entorno”, siendo su propósito central la provisión continua de "medios materiales” que satisfagan las necesidades de sustento de la sociedad. Como consecuencia, la estructura y la dinámica de la economía siempre están "arraigadas y enmarañadas en instituciones” (Ibíd.,: 34).

De acuerdo con Jessop (2008: 329), Polanyi quiso dar inicio al debate acerca de cómo buscar visiones alternativas a la existente economía capitalista, identificando en tal cometido tres principios de distribución distintos al del intercambio mediado por el mercado y orientado a la ganancia. En primer lugar, el principio de reciprocidad postula el intercambio entre grupos ordenados u organizados de manera semejante, a la vez que el principio de redistribución opera mediante un centro de asignación asociado a un régimen político. Finalmente, la modalidad doméstica householdingse basa en la producción orientada a la satisfacción de una unidad bastante autosuficiente, tal como "una familia, un asentamiento o un señorío” (1977: 3447; 1992: 35; 2001 [1944]: 47-53). En la economía real pueden coexistir dos o más principios, e incluso donde esté presente la ganancia monetaria no es del todo necesario que ésta sea dominante. Magali Sarfatti (2015) releva el alcance de lo señalado al afirmar que "la economía se institucionaliza de maneras tan distintas cuan distintas son las sociedades y su historia” (p.62)

La tarea conceptual de vertebrar una teoría social del desarrollo adecuada para las condiciones del mundo actual nos llama a no perder de vista las complejidades que resultan de las lógicas respectivas de los mercados, la política y la cultura, enfatizando su intensa interacción en las economías de mercado desarrolladas y en las en vías de desarrollo. El desafío en cuestión es captado con nitidez en las tres tesis principales de la teoría social neo-polanyiana, conforme a la sobresaliente versión de la misma desarrollada por Block (2012: 23-27).

Así, la primera tesis consigna que las economías de mercado siempre y en todo lugar se encuentran arraigadas socialmente. De manera nítidamente opuesta al "fundamentalismo de mercado" (Block y Somers, 2014: 3), 
a saber, la creencia exagerada y dogmática en la capacidad del libre mercado para resolver los problemas económicos y sociales, la tesis en cuestión redunda en que lisa y llanamente no existe la economía autónoma tal como se pretende. La economía ni siquiera analíticamente puede ser entendida como una entidad natural autorregulada. Pero también distanciándose de ciertos determinismos económicos del marxismo, Polanyi, en palabras de Block (2012) "prefirió una manera de pensamiento más complejo que veía los procesos económicos, los procesos culturales y los procesos políticos entremezclados de manera compleja"(p.21-22). En pocas palabras, nos encontramos ante el continuo arraigo de la economía y los mercados en la sociedad.

De este modo, la economía y los mercados se encuentran indefectiblemente arraigados en instituciones y arreglos político-estatales, ordenamientos legales, ideas y sistemas de creencias y conocimiento, prácticas culturales, etc. Todo ello, en su conjunto, incide significativamente en las lógicas y dinámicas de funcionamiento y creación de los mercados. Lo anterior refuerza nítidamente "la precedencia de la sociedad sobre la economía y confiere poder causal a la cultura”"2 (Sarfatti, 2015: 60).

A este respecto, es fundamental para la puesta en marcha y el funcionamiento de los mercados y la actividad económica en el capitalismo el hecho de que el trabajo, la naturaleza y el dinero, pese a no ser mercancías, sean tratadas como si lo fueran. Este paso fue absolutamente necesario para que "la búsqueda de provecho" se convirtiera en "el motor esencial del sistema económico” (Sarfatti, 2015: 62). De ahí la propuesta polanyiana de entenderlas como "mercancías ficticias” en rotunda oposición al fundamentalismo libremercadista que las concibe ilusoriamente como meras mercancías. Precisamente es el Estado el que interviene y modela significativamente tanto el carácter como la demanda y oferta de dichas “mercancías ficticias”. Por consiguiente, “el análisis de las mercancías ficticias destaca con particular claridad que la economía no existe sin el Estado ni fuera de la sociedad. Sirve, por tanto, para denunciar la gran ficción del pensamiento económico clásico” (Ibíd.,: 63).

A juicio de Polanyi (1982: 30; 2001 [1944]: 46-53), en las economías pre-capitalistas el proceso de producción estaba más o menos arraigado en diversas instituciones como la familia, el barrio, la comunidad, etc., mientras que en el capitalismo las relaciones económicas intentan ser extendidas a las "mercancías ficticias" del trabajo, el suelo y el dinero, con lo cual se aboga por una suerte de separación o "desarraigo" de las relaciones económicas capitalistas con respecto a las instituciones no económicas. Sin embargo, la economía simplemente no puede ser "desarraigada de su matriz sociocultural” (Sarfatti,2015: 62). Polanyi (2001 [1944]) sostiene que tal intento lisa y llanamente no puede prosperar del todo ya que: "Nuestra tesis es que la idea de un mercado completamente autorregulado es una completa utopía. Tal institución no podría durar sin aniquilar la sustancia humana y natural de la sociedad; destruiría físicamente al ser humano y transformaría su entorno en un desierto”. (p.3) 
La idea principal de la segunda tesis, a su vez, es que la sociedad de mercado y la economía mundial contemporánea son modeladas por un constante doble movimiento. El argumento consiste en que la doctrina del fundamentalismo de mercado, como se ha visto, es una auténtica "utopía” que, al intentar ser aplicada al pie de la letra, ocasiona efectos económicos, sociales y políticos altamente destructivos (Block y Somers, 2014: 9). En definitiva, su programa económico íntegro no puede ser implementado en plenitud. No obstante, al intentarse materializar el programa económico libremercadista, éste atiza al extremo la concepción formal de la economía capitalista, atentando, entre otras cosas, en contra de la integridad de las mercancías ficticias indicadas arriba, a saber, el trabajo, la naturaleza y el dinero. Dichas consecuencias activan de manera transversal y en sumo compleja una reacción social protectora, es decir, el segundo lado del doble movimiento.

Tal como subraya Block (2011): “un lado del doble movimiento es el esfuerzo por expandir el alcance de los mercados en la asignación de recursos y en la organización de la actividad económica... sin embargo, Polanyi también insiste en que el movimiento a favor de mercados expandidos genera un contramovimiento que busca proteger a la sociedad del mercado usando al gobierno para limitar y restringir el alcance de los mercados a la hora de proporcionar recursos directamente a los ciudadanos”.(p.24-25)

Lo anterior conduce a una situación paradojal en la que Estado no “pertenece” inequívocamente al mercado ni a la sociedad, ya que contradictoriamente sirve la dinámica del mercado, por una parte, y simultáneamente representa a la sociedad en contra del mercado, cristalizando la lucha y el conflicto entre ambos (Block y Somers, 2014: 64). Por una parte, el fundamento material de la sociedad supone depender de la sobrevivencia del mercado, con lo cual los intereses del mercado se convierten en intereses “generales", razón por la que al Estado no le queda espacio para no atenderlos, pero, por la otra, la clase trabajadora, amén de otras clases y grupos sociales, afectados negativamente por la expansión mercantil, bregan ante el Estado por protección social de manera transversal y defienden intereses generales para garantizar la sobrevivencia de la sociedad.

El pensador húngaro, conforme a Sarfatti (2015) no ve al Estado como “el comité ejecutivo de la burguesía”, sino más bien como el punto institucional en que se enfrentan los intereses generales de la sociedad. En la paradoja se expresa el “doble movimiento” polanyiano: “en una sociedad materialmente fundada sobre el mercado, tanto las leyes que protegen a sus víctimas como aquellas que favorecen la avanzada del mercado expresan intereses generales...Los Estados no sólo promulgan leyes que permiten mercantilizar a las no-mercancías, sino que también deben responder a los movimientos sociales que surgen en contra de la mercantilización: limitan, por lo tanto, su alcance mediante otras leyes y otras políticas, constituyendo así el doble movimiento que caracteriza a las sociedades de mercado” (p. 63). En síntesis, el Estado condensa la lucha contradictoria entre sociedad y mercado. 
Por último, la tercera tesis subraya que la contestación e impugnación política en múltiples niveles -local, regional, nacional y supranacionalda forma a los rumbos económicos disponibles a las sociedades en cualquier momento. Por cierto, de esta tesis se desprende un modelo de varios niveles de análisis que comprenden las lógicas de grupos y clases sociales dentro de una formación social, el papel político del Estado y el nivel o dimensión internacional o "global” de la economía y la política, dando cuenta de sus respectivos procesos institucionales (Block y Somers, 2014: 68-69).

La última tesis resulta fundamental, entre otras cosas, a efectos de aquilatar las posibilidades y constricciones del entorno económico y político global para las diferentes estrategias de desarrollo que se impulsen en América Latina, especialmente las de índole postneoliberal (Evans, 2008a; Coraggio, 2013; Cordera, 2013; Elizalde, 2013). De manera específica, Evans (2008a), desde premisas neo-polanyianas, ha elaborado un valioso abordaje a favor de la viabilidad de una posible "globalización contra-hegemónica" que supere al neoliberalismo. La idea central es propulsar un proyecto de transformación organizado a nivel global con el objetivo de potenciar sensiblemente el protagonismo político de instituciones, valores y prácticas democráticas, poniendo el acento en el desarrollo equitativo de las capacidades humanas y la sustentabilidad ecológica. Tal aporte conecta con importantes debates en curso en América Latina, que ponen el acento en cómo los esfuerzos por ampliar la democratización y explorar estrategias económicas alternativas al neoliberalismo en determinadas sociedades latinoamericanas enfrentan obstáculos y posibilidades en el actual contexto económico y político global (ver, por ejemplo, Coraggio, 2013; Cordera, 2013; Elizalde 2013).

\section{La “autonomía arraigada” y la propuesta de Block-Evans}

Como se ha indicado, en las últimas décadas la teoría social ha tendido a carecer de marcos conceptuales coherentes y sistemáticos acerca de las nuevas configuraciones entre Estado y economía. Por lo mismo, a continuación se ofrecerá una explicación del fenómeno aludido, al tiempo que se esbozará cómo la teoría social neo-polanyiana puede aportar significativamente a la elaboración de bases teórica solventes para el estudio de las relaciones entre Estado y economía.

La tesis de "traer de regreso al Estado" en algunos círculos de la ciencias sociales, en las décadas de 1970 y 1980, respondió fundamentalmente a la percepción de que los estudios políticos de la postguerra estaban excesivamente "centrados en la sociedad". De acuerdo con esta perspectiva, las funciones del Estado debían ser explicadas en términos de sus rasgos distintivos como un conjunto central de instituciones y organizaciones administrativas, o represivas, en lugar de ser entendidas como expresiones secundarias y derivadas de la sociedad. 
Por consiguiente, los teóricos neo-estatistas o "centrados en el Estado” de manera fundamental pusieron el acento en que el Estado y los “directivos estatales” podían contar con autonomía considerable frente a las presiones de grupos de interés (empresarios, sindicatos, gremios, etc.) insertos en el entorno social más amplio.

Pese al mérito de resaltar la importancia de los “directivos estatales” y del Estado en el desarrollo, el neo-estatismo terminó siendo presa de un estancamiento conceptual al no propiciar, en lo medular, un adecuado marco analítico que englobara las complejas relaciones entre el Estado, la economía y la sociedad civil (Block y Evans, 2007). En pocas palabras, el neoestatismo sólo visualizó la “autonomía” del Estado en desmedro de su “arraigo” o “incrustación” social.

Dada la problemática intelectual identificada arriba, corresponde de forma determinante al sociólogo Peter Evans (1995) dar finalmente con una notable formulación teórica que, incorporando ideas centrales de Polanyi, logra superar al neoestatismo en el campo de los estudios del desarrollo. Es menester insistir en la importancia de dicha contribución para propulsar la teoría social neo-polanyiana en América Latina y mejorar la comprensión de sus procesos de desarrollo.

Peter Evans (1995) coloca el énfasis en que la autonomía del Estado remite directa y sistemáticamente a su arraigo o incrustación en cruciales y diversos contextos y condiciones sociales. Así, Evans ha señalado que los Estados desarrollistas históricamente han contado con un sistema de toma de decisiones suficientemente efectivo que tiende a asemejarse relativamente al tipo ideal de la burocracia weberiana. La autonomía y el arraigo del Estado conforman la "base estructural subyacente" que da cuenta de las intervenciones de éste, así como de sus buenos resultados en materia de desarrollo económico (ibíd., 12).

Por consiguiente, el enfoque conceptual de la "autonomía arraigada” es una pieza decisiva para revitalizar la teoría social del desarrollo desde premisas neopolanyianas. A la fecha, la fertilización cruzada de ideas derivadas de Karl Polanyi ha llegado a su máxima expresión en el trabajo colaborativo de Fred Block y Peter Evans (Block y Evans, 2007).

La propuesta de Block y Evans (2007), cuenta con los siguientes méritos y promisorias pistas heurísticas para investigaciones posteriores. En primer lugar, se arguye que no hay fronteras claras y definitivas entre, por una parte, el aparato estatal y la sociedad, y, por la otra, los directivos estatales y las fuerzas sociales. Esto es un avance fundamental ya que el neoestatismo corría el riesgo de proponer que dado que el Estado y la sociedad estaban opuestos entre sí, cada uno podía ser estudiado aisladamente. Esto hubiera significado reificar y naturalizar distinciones sociales que son parciales y variables. Sin embargo, al rechazarse dicho supuesto, la distinción dicotómica, entre enfoques centrados en el Estado y centrados en la sociedad, simplemente pierde sentido. En otras palabras, el enfoque 
neoestatista falló fatalmente al insistir en entender el aparato estatal como variable independiente a la hora de explicar eventos políticos y sociales.

Otro mérito del enfoque Block-Evans es que abre un amplio espacio conceptual para comprender la separación del Estado de otros órdenes institucionales, entendiendo este proceso como socialmente construido y variable, en lugar de ser visto como algo natural. Además, tal separación permite conectar los órdenes institucionales y las fuerzas sociales a la vez que contener y separar a los directivos estatales de presiones de grupos sociales externos.

De la mayor importancia resulta ser que Block y Evans (2007) se abocan explícitamente al análisis neo-polanyiano de las sociedades en vías de desarrollo reforzando criterios y principios institucionales. Ambos autores brindan una valiosa síntesis de las corrientes institucionales, cuyo argumento central sostiene que el "Estado y la economía deberían verse como esferas de actividad que se constituyen mutuamente, ninguna de las cuales puede funcionar sin la otra” (Block y Evans, 2007: 308).

Siguiendo la noción polanyiana de arraigo social de la economía y los mercados, ambos teóricos sociales son enfáticos a la hora de afirmar que el hecho dialéctico fundamental es que "las economías de mercado se incorporan a una sociedad civil que se encuentra estructurada por el Estado y que la sociedad civil colabora a su vez a la estructuración de este último” (Block y Evans, 2007: 309).

La propuesta de Block y Evans (2007) debe ser enmarcada en la problemática investigativa en cuestión, al situar la innovación institucional para el desarrollo en el contexto de la sociedad civil, la cual, sin embargo, y a diferencia de las concepciones liberales de la misma, mantiene una activa y compleja interrelación de colaboración y tensión con el Estado.

Desde estas premisas Block y Evans,(2007) la sociedad civil comprende un conjunto de "actividades asociativas no gubernamentales", incluyendo, entre otras, las asociaciones empresariales, las organizaciones sindicales, los movimientos sociales, los partidos políticos y la "esfera pública” en la que la ciudadanía desarrolló sus "preferencias políticas". (p.309-310)

Paralelamente, los autores (Ibíd.: 310) ponen al descubierto las limitaciones de aproximaciones liberales que tienden a dar por hecho que el fortalecimiento de la sociedad civil y mayores niveles de "capital social" son suficientes para la solución de los problemas políticos y económicos. A modo de ejemplo, pese a que haya un fortalecimiento de la sociedad civil en un país, puede ocurrir que, no obstante, los grupos dirigentes de la sociedad se opongan tenazmente a la aparición y difusión de nuevas ideas y enfoques de política pública, trastabillando la innovación institucional y el consiguiente cambio social. Dicen Block y Evans (2007): "Las ideas firmemente arraigadas pueden cerrar el espacio político y reprimir el desarrollo de 
nuevas ideas. Es más, las elites políticas o económicas se resisten a menudo a cambiar las prácticas existentes, incluso si hay presiones considerables de la sociedad civil” (p.310)

Dado el arraigo de la economía y del Estado en la sociedad civil, resulta indispensable para el desarrollo que se promueva, en lugar de bloquear, la innovación institucional y de ideas, estructuras de conocimientos, visiones de sociedad, etc. De ahí que el desarrollo no pueda prescindir de una "sociedad civil viva, que contenga vínculos asociativos y concepciones normativas”, por la sencilla razón de que ésta “desempeña un papel central en el funcionamiento efectivo de la economía y del Estado” (Block y Evans, 2007: 310).

La lección analítica que se desprende del abordaje institucional de Block y Evans (Ibíd.: 311) es que el éxito en materia de desarrollo de los países obedece a una “capacidad de reconfiguración institucional”, a efectos de coordinar y articular específicamente entre sí el Estado, la economía y la sociedad civil, suponiendo "rutas institucionales múltiples hacia una economía exitosa y una gobernanza efectiva”, descartando la existencia de una "lógica puramente económica” a la que, sin embargo, se ha recurrido para justificar el “monocultivo institucional” (Ibíd.) propio del neoliberalismo.

\section{El "giro institucional"}

Pese a la importancia creciente que se atribuye a las instituciones y a sus interacciones, no contamos aún con definiciones compartidas de las mismas, ni tampoco con marcos analíticos ampliamente aceptados acerca de procesos humanos centrales que se dan en ellas (Miettinen, 2013; 2014).

Así entonces, la economía institucional concibe las instituciones como sistemas de reglas que crean un marco e incentivos para la toma de decisiones de los individuos. Por su parte, el institucionalismo histórico y social a menudo definen las instituciones en tanto que sistemas de reglas o sistemas que perduran debido a rituales, hábitos y rutinas que siguen sus integrantes. Tal concepto de institución, sin embargo, resulta ser demasiado estático para dar cuenta de la relación entre, por una parte, la continuidad histórica de las instituciones y, por el otro, el cambio y aprendizaje institucional (Miettinen, 2013).

Es así como el cambio y desarrollo de las instituciones se ha convertido en tema central tanto de la economía política (Mahoney y Thelen, 2010) como de la teoría organizacional neoinstitucional (Greenwood et al., 2002).

Comprender cómo se desarrollan, cambian y aprenden las instituciones constituye un desafío fundamental para el conocimiento y para las políticas y estrategias de desarrollo en un sentido amplio. Lo anterior conforma precisamente la base para la reconfiguración e innovación institucional analizada por Block y Evans (2007). 
En las dos últimas décadas los enfoques institucionales se han visto reforzados considerablemente en la sociología y los estudios organizacionales (Scott 2008a y 2008b; Djelic 2010). Conforme a esta perspectiva, las organizaciones se encuentran "arraigadas" en un contexto institucional o un campo organizacional (Di Maggio y Powell, 1983). Así, el campo multi-organizacional se ha convertido en el principal nivel de análisis para la aproximación institucional, haciendo posible el estudio de la interrelación entre las acciones organizacionales locales y las macroestructuras de la sociedad (Miettinen, 2013: 112).

En un primer momento, los estudios institucionales pusieron el acento en el "isomorfismo", es decir, en cómo y por qué las organizaciones de un campo tienden a ser homogéneas. La teoría económica neoinstitucional (Hodgson, 2004) tendió a concebir la continuidad y la reproducción como rasgos básicos de las instituciones. La explicación de lo anterior vendría dada por normas y estándares del Estado, organismos internacionales, asociaciones varias, así como por la regularidad y patrones de la conducta humana (Meyer, 2010).

El abordaje en cuestión con frecuencia entendió la estabilidad y la continuidad de las instituciones mediante patrones de comportamiento habitualizados y preconscientes de forma prácticamente mecanizada. Además de los patrones conductuales, el isomorfismo institucional fue explicado por los significados compartidos o los sistemas simbólicos culturales que son dados por sentados por los individuos socializados en las organizaciones de un campo determinado (Miettinen, 2013: 113).

Sin embargo, Miettinen (Ibíd.), indica que dicho enfoque no da cuenta debidamente de cómo cambian los hábitos y las rutinas, ni cómo la agencia individual y colectiva humana supone una "orientación activa hacia futuros alternativos" así como un "compromiso con proyectos transformadores”. En otras palabras, la agencia humana, en un sentido amplio, conlleva una perspectiva de “agencia distribuida” (Battilina y D’Aunno, 2009), donde ésta es estudiada preferentemente a la luz de cómo diferentes agentes contribuyen a procesos de cambios colectivos, al igual que en sus colaboraciones diversas, yendo más allá de un foco analítico exclusivo en el individuo.

En la actualidad, sin embargo, y como se ha afirmado, despunta un notable interés en el estudio del cambio, desarrollo y aprendizaje de las instituciones y las organizaciones (Miettinen 2013: 112), resaltando la distribución de prácticas emergidas localmente de un lugar a otro, así como la complejidad e incidencia de la agencia humana como dinámica central, subrayando la necesidad de abordar y poner al descubierto la transformación de las prácticas humanas (Ibíd.: 113).

Esta nueva perspectiva institucional (Ibíd.: 114) permite centrarse en la reconstrucción constante de las instituciones, en la experimentación local concomitante así como en el aprendizaje entre distintos actores (Sabel y Zeitlin, 2008; Thelen, 2010; Miettinen, 2013). 
Desafortunadamente, el reciente y marcado "giro institucional" en círculos intelectuales neoliberales de América Latina simplemente quedó a la zaga de los desarrollos conceptuales tratados arriba. La concepción neoliberal redunda en una empobrecida interpretación de las instituciones, las cuales quedan supeditadas a funciones de apoyo a lo que sería una lógica económica intrínseca de los mercados libres (ver Edwards, 2009). En tal perspectiva, la intervención económica del Estado es resaltada sólo en la medida que sea "amigable con el mercado", es decir, como mero apéndice o soporte para dinámicas económicas que supuestamente tienen lugar en mercados fundamentalmente autónomos. De ahí se desprende que, a juicio de Edwards (2009), cualquier alternativa económica y política al neoliberalismo del Consenso de Washington debe ser rechazada a priori por ser presa del "populismo". La lección de política pública brindada por el "giro institucional" es que las reformas de mercado de "primera generación" de las décadas de 1980 y 1990 tornan imperativo acometer reformas institucionales amigables con el mercado de "segunda generación”.

En suma, el institucionalismo neoliberal procura establecer que la economía de libre mercado, tras las dictaduras en varios países latinoamericanos, resulta indispensable en tanto soporte político de la recuperada democracia, a la vez que resultaba compatible con una fuerte orientación social en las políticas públicas (Edwards, 2009). De hecho, ésta es la interpretación preponderante que se hace del "modelo" chileno desde 1990 a la fecha para quienes dan por bueno el fundamentalismo de libre mercado (Garretón, 2013). Pero nuevamente los mercados y la economía no son entendidos como "arraigados" en la sociedad, ni en complejas relaciones de interdependencia. Tampoco se consideró seriamente el alcance potencialmente beneficioso de las intervenciones del Estado en la economía. En otras palabras, esta manera de pensar y su recetario de políticas simplemente no rompió con el supuesto conceptual de la "primacía de la producción”, que caracteriza indefectiblemente al pensamiento neoliberal, permaneciendo indiferente, por ejemplo, a la valiosa propuesta de la "autonomía arraigada" del Estado. De la mayor importancia a este respecto es que se perdió de vista la centralidad de la "innovación institucional" defendida por Block y Evans (2007), y que es vital para acometer auténticas "reformas de segunda generación” en lugar de sus empobrecidas versiones neoliberales.

\section{El Estado Desarrollista Red y América Latina}

Los argumentos neo-polanyianos también son de alta relevancia actual para una mejor comprensión de distintos problemas económicos y políticos que enfrenta el desarrollo en América Latina.

En tal perspectiva, Evans (2007, 2008b) ha proseguido la elaboración conceptual sobre cómo deberá entenderse tanto el desarrollo como el papel del nuevo Estado desarrollista en el mismo en las primeras décadas del siglo XXI. Por consiguiente, hay nuevas condiciones, dinámicas y concepciones acerca de ambos fenómenos que redundan en la creciente importancia 
de la innovación, la educación, las instituciones, el capital humano y las capacidades, etc., en el desarrollo y el Estado por igual, con lo cual pierden vigencias la formas convencionales de entender la problemática aludida (Evans, 2008b).

Una manera de seguir la pista indicada por Evans, es entender al nuevo "Estado Desarrollista Red” como expresión de lo indicado arriba, en contraposición al convencional "Estado desarrollista" de décadas pasadas. Llama la atención que las dificultades y escollos para ahondar en la comprensión de la transformación en cuestión no sólo se encuentra en el pensamiento económico y social neoliberal, sino que también en corrientes críticas que ignoraron la relevancia permanente de la economía política y del Estado. Por consiguiente, el Estado no es mero reflejo o registro de lo que ocurre en la economía, o un obstáculo pernicioso para el desarrollo, y debe ser analizado en sus propios términos, siendo incluso actor estratégico para el funcionamiento dinámico de la economía (Mazzucato, 2013).

No obstante, al igual que los mercados, los Estados desarrollistas, tal como se ha reiterado, no existen ni operan como si estuvieran por encima o fuera de la sociedad. Así entonces, y desde premisas neo-polanyianas, se debe evitar la tendencia a concebir dicho Estado como un dispositivo que prescinde mayormente de la sociedad, asumiendo el papel de una suerte de "sujeto técnico" de promoción del desarrollo.

La activa innovación institucional y la transformación del Estado a las que se ha hecho mención reiteradamente exigen cambios conceptuales importantes para dar cuenta debidamente del nuevo Estado desarrollista, especialmente en el ámbito de la economía de la innovación y el impulso tecnológico (Block, 2008; Block y Keller, 2011).

En efecto, hay en curso una vigorosa línea de investigación sobre el “Estado Desarrollista Red” (EDR), que documenta y desmenuza su intervención activa en la "economía de la innovación” de países que lideran en el traspaso del avance científico-tecnológico a las empresas y la competitividad económica (Block, 2008; Block y Keller, 2011; ÓRiain, 2004). Por ende, la teoría social neo-polanyiana ofrece un notable soporte conceptual a las formulaciones acerca del Estado Desarrollista Red con las más variadas ramificaciones e implicancias tanto para las economías de mercado desarrolladas como para las en vías de desarrollo.

En evidente contraste con el Estado desarrollista de los países del sudeste asiático, especialmente entre las décadas de 1950 y 1980, cuyas formas de organización eran más bien jerárquicas, centralizadas y burocráticas, el nuevo Estado desarrollista en los EE.UU., Escandinavia, Irlanda, etc., se caracteriza por la creciente adopción de modalidades de organización en red más adecuadas a entornos económicos y sociales de alta complejidad. En estos entornos complejos, el EDR, pese a contar con autonomía, se incrusta en la sociedad civil y los mercados de tal modo de propiciar sinergias positivas entre ellos (Block y Evans, 2007). 
Lo consignado hace posible poner el acento en el hecho de que la innovación, en las regiones más dinámicas del mundo, se sustenta a menudo en redes de instituciones descentralizadas. Asimismo, la finalidad de dichas instituciones ha solido ser la construcción de comunidades de empresas e instituciones en lugar de la promoción de la empresa individual líder, que definía la anterior política tecnológica del estatismo desarrollista.

El papel del EDR es indispensable para la economía de la innovación, puesto que asume iniciativas de liderazgo y numerosos riesgos para poner en marcha las redes que conectan las empresas entre sí (Mazzucato, 2013). Asimismo, frente al problema de "fallas de red", el Estado cumple tareas clave en orden a reducir el oportunismo y la falta de capacidades de los actores empresariales que se desenvuelven en las mismas (Whitford y Schrank, 2011).

De acuerdo con Block (2008: 172-174), el EDR fija como objetivo fundamental el logro de la competitividad económica estimulando la productividad de los ingenieros y los científicos existentes en la sociedad. Para tales efectos, el EDR lleva a cabo un conjunto de acciones estatales y de política pública de manera altamente descentralizada. Esto significa, a modo de ejemplo, que sus múltiples acciones no tienen un único centro alojado en alguna organización específica ya que éstas, por el contrario, se despliegan en un gran número de organizaciones e instituciones del aparato estatal.

De forma decisiva para América Latina, la literatura acerca del EDR enfatiza que no hay razones para descartar que las estructuras y estrategias del EDR también puedan abrirse paso y prosperar en algunos países de la región, donde, pese a su modesto nivel de desarrollo y avance científicotecnológico, sí es factible lograr progresos significativos en el mejoramiento industrial ${ }^{3}$ ("industrial upgrading”) (Negoita y Block, 2012; Pérez, 2008). Por supuesto, la inevitable aparición de fallas y limitaciones en el funcionamiento de las redes requerirá de un activo “emprendimiento” estatal (Ibíd.; Mazzucato, 2013).

El cariz emprendedor del papel del Estado (Mazzucato, 2013), en la colaboración público-privada, constituye una ayuda inestimable para las empresas de las naciones avanzadas al momento de reducir la incertidumbre y complejidad del entorno económico global. De manera semejante, la experiencia exitosa de empresas chilenas del sector industrial agroexportador, especialmente a contar de la recuperación de la democracia en 1990, ilustra meridianamente cómo organizaciones y programas estatales han sido decisivos para la creación de redes empresariales y la superación de "fallas de red" (Block y Negoita, 2012). Las empresas chilenas en cuestión se han beneficiado de la formación de redes entre ellas, con lo cual han podido llevar a cabo estrategias organizacionales que distribuyen los notables riesgos asociados a la adopción de nuevas tecnologías, al tiempo que han mejorado colectivamente su aprendizaje y conocimiento.

El caso chileno ilustra el decisivo potencial del EDR para las socie- 
dades latinoamericanas en ciertos nichos de exportación agroindustrial, pese a no disponer de capacidades económicas y científico-tecnológicas comparables a las de las de sociedades más avanzadas.

Empero, la estructura productiva y la economía chilena aún siguen atrapadas por el neoliberalismo en ámbitos clave (ver, por ejemplo Garretón, 2013; Mayol, 2012). El predominio neoliberal conlleva que, entre otras cosas, el EDR se mantenga invisibilizado en la esfera pública, prevaleciendo el discurso gerencialista y tecnocrático de las elites económicas y políticas, que ensalza el emprendimiento privado e individualista, así como las supuestas bondades del libre mercado libre. Este fenómeno se asemeja considerablemente a lo documentado en esta materia por ÓRiain (2011) y Block (2008) para los casos de Irlanda y los EE.UU. respectivamente. Por tanto, es menester identificar los numerosos obstáculos neoliberales que la sociedad chilena deberá superar si es que pretende contar con una estrategia nacional de política industrial (Rodrik, 2004) que, desde lo logrado hasta ahora por su EDR, haga posible sentar las bases para una auténtica economía del conocimiento y la innovación (Negoita y Block, 2012; Agosín y Bravo, 2007).

Lo anterior, cómo no, amplía sensiblemente el análisis del desarrollo hacia desafíos que, como señala Santos (2005, 2009), conllevan la enorme tarea de profundizar radicalmente la democracia y el Estado en una perspectiva post-neoliberal, así como la reconceptualización en América Latina de la “emancipación social” (2009). Tal idea entronca de lleno con la visión de Polanyi en torno a la indispensable "nueva filosofía pública”. Por cierto, lo anterior presupone también cambios significativos a nivel global que amplíen el abanico de políticas públicas disponibles para los gobiernos de los países en vías de desarrollo (Evans, 2008a).

Para tales desafíos, en el ámbito teórico, son de la mayor importancia varias ideas y argumentos neo-polanyianos que han sido expuestos arriba sobre cómo entender las relaciones entre Estado y economía en el desarrollo, el papel clave de una sociedad civil viva, la dinámica de la innovación institucional y, por último, el potencial del Estado Desarrollista Red. En síntesis, la teoría social neo-polanyiana ofrece herramientas analíticas para hacer inteligibles tales fenómenos y procesos, contribuyendo de manera significativa al estudio del desarrollo en América Latina.

\section{Conclusión}

A lo largo de la exposición precedente de la teoría social neopolanyiana, se ha procurado ofrecer una interpretación sistemática de sus principios e ideas centrales, al tiempo que se ha indicado cómo hacerla relevante para el estudio de procesos político-económicos y sociales de América Latina. Dicha orientación supone la apertura a un diálogo crítico con diversos debates en curso en América Latina. Como se ha visto, hay valiosas lecciones de la perspectiva neo-polanyiana tanto para una mejor 
comprensión del neoliberalismo en el subcontinente, así como para los distintos esfuerzos a favor de estrategias alternativas y democráticas postneoliberales. Por lo tanto, se ha intentado indicar cómo las premisas neo-polanyianas arrojan luz acerca de las relaciones entre Estado y economía. Simultáneamente, se ha puesto de manifiesto la utilidad de la teoría social neo-polanyiana para el actual estudio del desarrollo. Por último, el enfoque esbozado se ha centrado concretamente en el debate sobre el emergente Estado Desarrollista Red, su parcial plasmación en Chile, y en las posibilidades que podría ofrecer a las sociedades latinoamericanas contemporáneas en una orientación post-neoliberal. 


\section{Notas}

${ }^{1}$ Las ideas y conceptos de Karl Polanyi han sido discutidos en otras recientes formulaciones, entre las cuales, por ejemplo, cabe destacar el "enfoque estratégicorelacional” de B. Jessop (2008), quien sugerentemente relaciona dichas ideas con el enfoque sistémico de Luhmann y la teoría de la regulación. Sin embargo, el presente artículo releva el trabajo de teóricos que rescatan recursos conceptuales adicionales en el pensamiento de Polanyi que no se encuentran en toda su riqueza, profundidad y alcance en otros marcos analíticos.

${ }^{2}$ El notable trabajo teórico de J. L. Coraggio (2009) relaciona ideas y conceptos de Karl Polanyi con los importantes debates latinoamericanos sobre la economía social y solidaria.

${ }^{3}$ De manera altamente relevante para el desarrollo democrático de América Latina, Carlota Pérez pone el acento en los requisitos e innovaciones institucionales y organizacionales, amén de la necesidad de contar con un nuevo pensamiento social, en tanto que condiciones indispensables para que prospere cabalmente la revolución tecnológica en curso (Pérez, 2002, 2008). 


\section{Bibliografía}

Block, F. (2008), "Swimming against the current: the Rise of a Hidden Developmental States in the United States” enPolitics\&Society 36 (2), p.169206.

Ídem (2011), “Contesting markets all the way down” en Journal of Australian Political Economy, (68), 27.

Agosin, M. y Bravo, C. (2007), The emergence of new successful export activities in Chile en Latin American Research Network, Inter-American Development Bank, Washington, DC.

Battilana, J., y T. D’Aunno. (2009), “Institutional Work and the Paradox of Embedded Agency” en Institutional Work: Actors and Agency in Institutional Studies of Organizations. Cambridge UniversityPress, Cambridge, U.K. p. 31-58.

Block, F. y Evans, P. (2007), “El Estado y la economía”, en Instituciones y desarrollo en la era de la globalización neoliberal. ILSA, Bogotá, p. 307-350.

Block, F. y Keller, M. (eds.) (2011), State of innovation. The U.S. Government's role in Technology Development, Paradigm Publishers, Boulder.

Block, F. y Somers, M. (2014), The Power of Market Fundamentalism. Karl Polanyi’s Critique, Harvard University Press, Boston.

Blyth, M. (2015), Austerity. The history of a dangerous idea. Oxford UniversityPress, Oxford.

Coraggio, J. (2009), “Polanyi y la economía social y solidaria en América Latina” en J.Coraggio (Org) ¿Qué es lo económico? Materiales para un debate necesario contra el fatalismo, Editorial Ciccus, Buenos Aires, p. 109-168.

Ídem, (2013), “Otra economía, otra política, otra izquierda”, en América Latina en Movimiento 482: 1-5.

Coraggio, J., Caillé, A., Laville, J., y Cyrille, F. (2009). ¿Qué es lo económico? Materiales para un debate necesario contra el fatalismo, EditorialCiccus, Buenos Aires.

Cordera, R. (2013), “Utopías realizables: algunas hipótesis de trabajo”América Latina enMovimiento 482, p.10-15.

Di Maggio, P. y Powell, W. (1983), “The Iron Cage Revisited: Institutional Isomorphism and Collective. Rationality in Organizational Fields” en American Sociological Review 48 (2), p.147-160. 
Djelic, M. (2010), “Institutional Perspectives-Working towards Coherence or Irreconcilable Diversity?” enOxford Handbooks Online, p. 15-39.

Edwards, S. (2009), Populismo o mercados: el dilema de América Latina. Editorial Norma, Bogotá.

Evans, P. (1995), Embeddedautonomy, Princeton UniversityPress: Princeton.

Ídem, (2007), Instituciones y desarrollo en la era de la globalización neoliberal, ILSA, Bogotá.

Ídem, (2008a). “Is an alternative globalization possible?” in Politics \& Society, 36(2), p.271-305.

Ídem (2008b), "In search of the 21st century developmental state" in The Centre for Global Political Economy, University of Sussex Working Paper, 4.

Friedman, M. y Friedman, R. (1982), Libertad de elegir. Hacia un nuevo liberalismo económico, Grijalbo, Barcelona.

Garretón, M. (2013), Neoliberalismo corregido y progresismo limitado: Los gobiernos de la Concertación en Chile, 1990-2010, Editorial ARCIS, Santiago.

Greenwood, R., Suddaby, R. y Hinings, C. R. (2002), “Theorizing change: The role of professional associations in the transformation of institutionalized fields”. Academy of Management Journal, 45, p. 58-80.

Hodgson, G. (2004), The Evolution of Institutional Economics: Agency, Structure and Darwinism in American Institutionalism. Routledge, London.

Mahoney, J., y Thelen, K. (2010), “A Theory of Gradual Institutional Change” in Explaining Institutional Change: Ambiguity, Agency, and Power. Cambridge UniversityPress, Cambridge, p. 1-37.

Mayol, A. (2012), El derrumbe del modelo: la crisis de la economía de mercado en el Chile contemporáneo, LOM Ediciones, Santiago.

Mazzucato, M. (2013), The entrepreneurial state: Debunking public vs. private sector myths, Anthem Press.

Meyer, R. (2010), “New sociology of knowledge: Historical legacy and contribution to current debates in institutional research" en The Sage Handbook of Organizational Institutionalism, Sage, Los Angeles, p.519-538.

Miettinen, R. (2012). Innovation, human capabilities, and democracy: Towards an enabling welfare state. Oxford University Press, Oxford. 
Ídem (2013), Innovation, Human Capabilities, and Democracy: Towards an Enabling Welfare State, University Press, Oxford.

Ídem (2014), “Information technological revolution and institutional innovations” en Center for Research on Activity, Development and Learning. Working papers 4/2014, University of Helsinki, Helsinki.

Negoita, M., y F. Block (2012), "Networks and Public Policies in the Global South: The Chilean Case and the Future of the Developmental Network State” en Studies in Comparative International Development 47 (1), p. 1-22.

Ó Riain, S. (2004), The Politics of High-Tech Growth: Developmental Network States in the Global Economy. Cambridge University Press, Cambridge.

Perez, C. (2002). Technological revolutions and financial capital: The dynamics of bubbles and golden ages. Edward Elgar Publishing, Londres.

Ídem (2008), “Una visión para América Latina: dinamismo tecnológico e inclusión social mediante una estrategia basada en los recursos naturales” en Revista Económica, 14(2).

Polanyi, K. (1977). The livelihood of man, Academic press, New York.

Ídem (1982), “The economy as instituted process. Trade and market in the early empires” in Granovetter, M. \&Swedberg, R. (Eds.) The sociology of economic life. Westview Press.

Ídem (2001) [1944], The Great Transformation. The political and economic origins of our time. Ameron House, Nueva York.

Rodrik, D. (2004), Industrial policy for the twenty-first century.CEPR Discussion Paper No. 4767. Harvard University.

Sabel, C.F. y Zeitlin, J. (2008), “Learning from Difference: The New Architecture of Experimentalist Governance in the EU” en European Law Journal, Vol. 14, Issue 3, p. 271-327.

Santos, B. (2005), Reinventar la democracia. Reinventar el Estado, CLACSO,Buenos Aires.

Ídem (2009), Pensar el Estado y la Sociedad: Desafíos actuales, CLACSO/ Waldhuter, Buenos Aires.

Sarfatti, M. (2015), “El fundamentalismo de mercado o cómo dura una ideología” en Revista Argumentos, Edición N 1, Año 9.

Scott, W. (2008a), Institutions and Organizations: Ideas and Interests(3rd ed.), Sage publications, Los Angeles. 
Ídem (2008b), “Approaching adulthood: the maturing of institutional theory” en Theory and society 37, p. 427-442.

Thelen, K. A. (2010), "Beyond Comparative Statics: Historical Institutional Approaches to Stability and Change in the Political Economy of Labor" en The Oxford Handbook of Comparative Institutional Analysis, Oxford University Press, Oxford, p. 41-61.

Wade, R. (1990), Governing the Market, Princeton University Press, Princeton.

Whitford, J., y Schrank, A. (2011), "The paradox of the weak state revisited: industrial policy, network governance, and political decentralization” In State of innovation: The US government's role in technology development, p.261-281. 